\title{
Children's Quality of Life in Europe: National Wealth and Familial Socioeconomic Position Explain Variations in Mental Health and Wellbeing-A Multilevel Analysis in 27 EU Countries
}

\author{
Ulrike Ravens-Sieberer, ${ }^{1}$ Hana Horka, ${ }^{2}$ Agnes Illyes, ${ }^{3}$ Luis Rajmil, ${ }^{4,5,6}$ \\ Veronika Ottova-Jordan, ${ }^{1}$ and Michael Erhart ${ }^{1}$ \\ ${ }^{1}$ Research Unit Child Public Health, Department of Child and Adolescent Psychiatry, Psychotherapy, and Psychosomatics, \\ Center for Psychosocial Medicine, University Medical Center Hamburg-Eppendorf, 20246 Hamburg, Germany \\ ${ }^{2}$ European Commission, Health and Consumer DG, C4-Health Determinants, 2920 Luxembourg, Luxembourg \\ ${ }^{3}$ Gallup Organization, Regional Headquarter for CEE Hungary, 1033 Budapest, Hungary \\ ${ }^{4}$ Agència de Qualitat i Avaluació Sanitàries (AQuAS), 08005 Barcelona, Spain \\ ${ }^{5}$ Health Services Research Group, IMIM (Hospital del Mar Medical Research Institute), 08003 Barcelona, Spain \\ ${ }^{6}$ The Biomedical Research Centre in Epidemiology and Public Health (CIBERESP), 28029 Madrid, Spain
}

Correspondence should be addressed to Ulrike Ravens-Sieberer; ravens-sieberer@uke.de

Received 30 August 2013; Accepted 17 October 2013

Academic Editors: C. C. Branas, N. Kontodimopoulos, B. Polivka, and A. Zaborskis

Copyright (C) 2013 Ulrike Ravens-Sieberer et al. This is an open access article distributed under the Creative Commons Attribution License, which permits unrestricted use, distribution, and reproduction in any medium, provided the original work is properly cited.

Sizeable variations in quality of life (QoL) and wellbeing according to socioeconomic status and national wealth have been observed. The assessment of children's wellbeing may vary, depending on whether a parental or a child perspective is taken. Still, both perspectives provide important and valid information on children's wellbeing. The Flash Eurobarometer no. 246 which was conducted for the European Commission assesses parents' reports on their children's health and wellbeing in $27 \mathrm{EU}$ member states. Overall, 12,783 parents of 6-17-year-old children in the $27 \mathrm{EU}$ states participated in telephone interviews. Parents reported children's QoL and wellbeing using the KIDSCREEN-10 measure, as well as their occupational status and education level. Within a multilevel analysis, the KIDSCREEN-10 was regressed on parental occupation and education level. Random intercepts and slopes were regressed on gross domestic product per capita and income inequality. Low QoL was reported in 11.6\% of cases, whereby crossnational variation accounted for $13 \%$ of the total variance in QoL. Children from countries with higher national wealth and lower income inequality were at lower risk for low QoL and wellbeing. Higher age of the child, a medium or low parental occupational status, and low parental educational status were associated with a higher risk for low QoL and wellbeing.

\section{Introduction}

It is commonly reported that a higher socioeconomic status (SES) is associated with better health in adults, children, and adolescents [1]. Previous studies in adolescents have found associations between socioeconomic status and indicators of mortality and morbidity as well as self-rated health [2-10].
Subjective health and wellbeing has been increasingly recognised as important for predicting health services utilisation and future morbidity. It has also been increasingly recognised as important for detecting hidden morbidity and health care needs that are not identified using traditional medical indicators [11-14]. Subjective health, or quality of life (QoL) [11], has been defined as "individuals' perceptions of 
their position in life in the context of the culture and value systems in which they live and in relation to their goals, expectations, standards, and concerns." QoL and wellbeing incorporates a person's physical health, psychological state, level of independence, and social relationships, as well as personal beliefs and their relationships to salient features of the environment [15].

Socioeconomic status concerns aspects of education, occupation status, and income. It has been argued that the relation between SES and health is due to both a psychosocially mediated general susceptibility of the disadvantaged and reactions to material and psychosocial stress inducing conditions [10]. In addition, aspects of nutrition, health behaviour habits, unhealthy housing and living situations, and access to services and social resources, as well as knowledge about health and health risk factors have been discussed. Some studies support the hypothesis that behavioural factors such as smoking, sedentary behaviours, and nutrition partly account for the association between family material wealth and adolescents' self-rated health and wellbeing [2].

The association between population health and population wealth is also well established [16]. Significant variations in the level of material wealth might affect the general quality of housing, access to health care, and education $[17,18]$. Income inequalities have also been considered; perceptions of place in the social hierarchy cause psychosocial processes such as negative emotions, shame, and distrust [19]. This could foster antisocial behaviour and reduce civic participation which could ultimately lead to less social capital and cohesion within the community [20].

Few studies have measured social inequalities in the health and wellbeing of children and adolescents on both the individual and population level [2, 18, 21]. Multilevel effects [22] of social deprivation have been found at both the individual and national levels [23]. However, previous studies mainly analysed categorical and ordinal outcomes, which preclude parametric estimation of the proportion of variance attributable to individual socioeconomic factors and national wealth. The cross-national comparability of most of these health indicators is also unclear.

This paper aims to assess differences in parental ratings of their children's QoL and wellbeing in the 27 member states of the European Union. The impact of national wealth and income inequalities as well as parental occupational and educational status on the children's QoL and wellbeing are examined using the KIDSCREEN-10 Index of QoL and wellbeing which is a measure that, to the best of our knowledge, provides interval-scaled and cross-national comparable health information $[24,25]$.

\section{Methods}

2.1. Design and Procedures. The Flash Eurobarometer "Parents' views on the mental health of their child" (no. 246) survey was conducted for the European Commission, European Commission Health and Consumers DG, and C4Health Determinants. Telephone interviews were conducted in September 2008 by several national institutes under the leadership of the European Gallup Institution. The target population was randomly selected parents (including stepparents/guardians) of children 6-17 years old in each of the EU 27 member states. The target (and actual) national sample size was 500 respondents (250 respondents each in Cyprus, Malta, and Luxembourg) [26].

2.2. Instruments and Variables. The KIDSCREEN-10 is a valid and reliable measure [25] which assesses the previous week's affective symptoms (depressed mood), cognitive symptoms (disturbed concentration), psychovegetative aspects (vitality, energy, and feeling well), and psychosocial aspects of QoL and wellbeing (the ability to experience fun with friends, relation with parents, and getting along well at school). The instrument is available in all European languages and was applied in the national language in each country. Item answers were (re-)coded so that higher values indicated better wellbeing. The sum score was transformed into a Rasch score [27], with a mean of 50 and a standard deviation of 10 . Respondents with a score -1.2 SD below the mean (i.e., below 38) were classified as "noticeably low wellbeing" [24].

Parents were asked about the age, gender, and health of one eligible child with the birthday nearest to the interview date. The responding parents' occupation status was assessed in the categories "low" (farmer, forester, fisherman, manual worker, or unskilled manual worker), "medium" (owner of a shop, craftsman, or other), and "high" (professional lawyer, medical practitioner, accountant, architect, general or top management position, or manager of company). Education status of the responding parent was assessed in the categories "low" (finished full-time education at 17 years or younger or never been in full-time education), "medium" (finished at age 18-24), and "high" (finished at age 25 or older) [26].

Macroeconomic variables included the gross domestic product (GDP) per capita at purchasing power parity (PPP) (in thousands of Euros) and the Gini Index of income inequality. Both indicators were estimated for the year 2008 $[28,29]$.

The GDP indicates the total market value of all final goods and services produced in a country in a given year, which is equal to total consumer, investment, and government spending, plus the value of exports, minus the value of imports. Countries' PPP takes into account the cost differences across countries of buying a similar basket of goods and services in numerous expenditure categories, including nontradables [28].

The Gini Index is based on the "Lorenz curve." The Lorenz curve plots the cumulative percentage of household income on the vertical axis against the cumulative percentage of households on the horizontal axis. The Gini Index is defined as the area between the line of perfect equality and the observed Lorenz curve as a percentage of the area between the line of perfect equality and the line of perfect inequality [29].

2.3. Statistical Analyses. Linear multilevel regression analysis [22] was performed using the software HLM 5.05 [30]. Multilevel analyses account for the fact that respondents 
within a certain context (e.g., country) may be more similar to each other than to individuals from a different context (i.e., intracontext correlation). On the other hand, the variability of effects across different contexts is estimated and the role of contextual factors for this variability can be studied [22].

A hierarchical two-level model including separate variances at the individual and at the country level was specified with random variation of the intercepts and slopes across countries. Beforehand, the reliability of the cross-country variation was examined: an intraclass correlation coefficient $>0.10$ indicated that at least $10 \%$ of the observed crosscountry variation in the coefficients represented "true" differences. Such coefficients (intercepts and slopes) were specified as "random" and the coefficients and their distribution of variation across countries were estimated. The variance partitioning coefficient (VPC) denotes the proportion of "reliable" variance in the outcome that is attributable to country differences. In line with the recommendations of Cohen, a proportion of 0.14 was classified as a large effect [31].

The KIDSCREEN-10 scores were regressed on the child's age and gender, parental occupational status, and educational status. The analyses were controlled for parental gender. The random variation of the intercepts and the slopes were regressed on the gross domestic product per capita at purchasing power parity in thousands of Euros (GDP) and the UN Gini Index of income inequality in the EU 27 countries. The analyses were then repeated for the dichotomised KIDSCREEN-10 scores employing multilevel logistic regression analysis. Analyses were repeated across age groups 6-10, 11-14, and 15-17 years as well as for male and female reporting parents.

The actual sample size of $n=500$ cases per country enabled us to detect a small effect $\left(R^{2}=0.03\right)$ in the association between individual predictors and QoL with a power of $P=0.80$ (at an alpha level of $P=0.05$ ) when running a linear regression analysis with nine predictors.

Due to the absence of systematic information regarding the sociodemographic and economic characteristics of such a specific parent population (with children aged 6-17 years) in some countries, a nonresponse weighting was not carried out.

\section{Results}

3.1. Sample Characteristics. Table 1 shows that the majority of responding parents were female. The proportion of girls ranged from $45.6 \%$ (Slovenia) to $53.0 \%$ (Austria). The GDP ranged from 11.1 for Romania to 79.4 for Luxembourg. The largest income inequality emerged for Portugal (Gini $=38.5)$ and the smallest was reported for Denmark (Gini $=24.7)$.

3.2. Basic Questionnaire Characteristics. Table 2 shows that the items in the KIDSCREEN-10 instrument were well fitted to the Rasch Partial Credit Model, with Infit MSQ values between 0.7 and 1.3. Mean KIDSCREEN-10 scores varied between 43.1 (Estonia) and 57.9 (The Netherlands). These differences were also reflected in the percentages of respondents

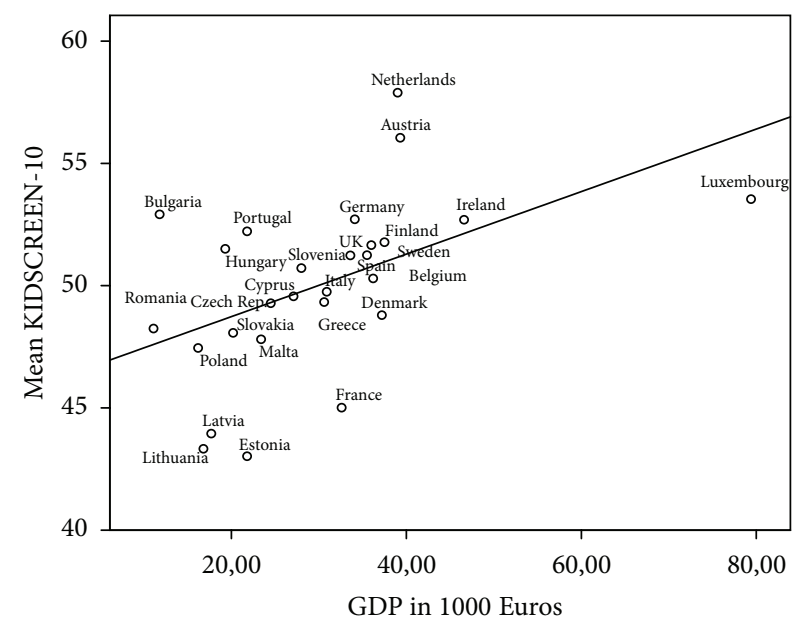

FIGURE 1: National wealth (GDP) and mean HRQoL ratings (KIDSCREEN-10). The diagonal line refers to the regression line for national mean KIDSCREEN-10 on country GDP $\left(R^{2}=0.239\right)$.

with noticeably low scores $(<38)$, ranging from $1.9 \%$ (The Netherlands) to $29.5 \%$ (Estonia).

\subsection{Socioeconomic Inequalities in Health}

3.3.1. Raw Analysis. We found large cross-national variation in QoL and wellbeing ratings for the children. Figure 1 shows that higher GDP was associated with higher mean KIDSCREEN-10 ratings. A linear regression analysis of mean KIDSCREEN-10 scores and country GDP across countries resulted in a coefficient of determination of $R^{2}=0.24$.

3.3.2. Linear Regression Analysis. Table 3 shows the multilevel linear regression results for the KIDSCREEN-10 scores. For the analyses on the individual level, the reference group consisted of 6- to 10-year-old boys whose reporting parent was male with a high occupational status and education finished at age 17 years or older. The KIDSCREEN-10 point estimate for this group was 52.3 (51.1-53.5).

Compared with the reference group, older age was associated with a mean decrease of as much as 1.7 (1.6) points in the KIDSCREEN-10 for 11- to 14-year-old boys (girls) and 2.8 (2.5) points for 15- to 17-year-old boys (girls) in subjective health ratings on the KIDSCREEN-10 (numbers in parentheses refer to girls). Compared to a high occupational status, a low (medium) occupational status of the reporting parent was associated with a decrease of $1.4(0.5)$ points in the KIDSCREEN-10. Low educational status resulted in a 0.8 point decrease in the KIDSCREEN-10.

At country level, the variance partitioning coefficient (VPC) indicated that $13.0 \%$ of the total variance in the KIDSCREEN-10 was attributable to "true" country differences. Taking Cohen [31] into account, such a percentage resembles a nearly "large" effect size. For $95 \%$ of all comparable countries, the estimated mean of the KIDSCREEN10 scores lay between 46.7 and 57.9 points. About $13.5 \%$ of that cross-national variation was attributable to the national 
TABLE 1: Sociodemographic and socioeconomic characteristics of the sample.

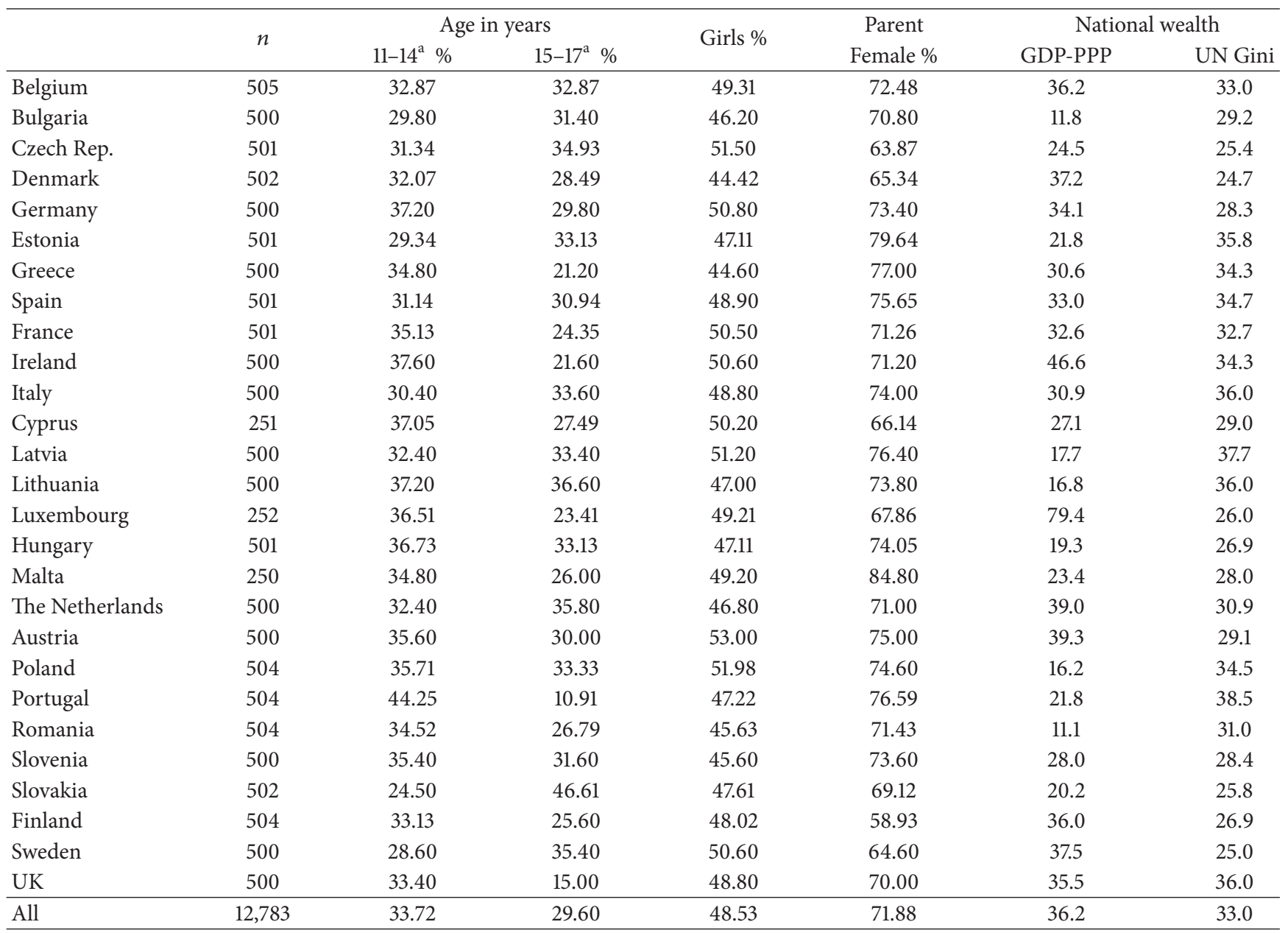

add up to $100 \%$ with $6-10$-year olds;

GDP-PPP: Gross domestic product per capita at purchasing power parity in 1000 Euros;

UN Gini: United Nations Index of income inequality.

wealth and income inequalities: a rise of ten thousand Euros in GDP per capita was associated with an increase of 0.9 in the KIDSCREEN-10. An income inequality increase of one point on the Gini Index was associated with a 0.22 point decrease in KIDSCREEN-10 score. In total, GDP and Gini income inequality accounted for $1.8 \%$ of the total variation (individual and country level) in subjective health. The decreasing effect of being a 15- to 17-year-old girl varied across countries. It was estimated that in $95 \%$ of all comparable countries this effect ranged from -4.3 to -0.7 points. The effect of gender of the reporting parent also varied across cultures. All other predictor variables (e.g., parental occupation and parental education) displayed only small and statistically nonsignificant cross-country variability in the magnitude of their effects on QoL and wellbeing.

3.3.3. Logistic Regression Analysis. Table 4 shows that higher age increased the "chance" of noticeably low QoL (KIDSCREEN score < 38) by up to 1.8 (1.5)-fold for 11- to 14-yearold boys (girls) and by up to 2.2 (2.0)-fold for 15- to 17-yearold boys (girls). Compared with a high occupational status, a low occupational status of the reporting parent was associated with a 1.4-fold higher chance of a noticeably low QoL and wellbeing. A medium occupational status was still associated with a 1.2-fold higher chance of a noticeably low QoL and wellbeing. Low educational status of the parents increased the risk (OR) of a noticeably low QoL and wellbeing score by 1.3 .

At country level, the base risk (of the reference group) for a noticeably low KIDSCREEN-10 outcome varied across countries. It was estimated that for $95 \%$ of all comparable countries the base risk was 0.02 to 0.16 . This risk decreased by $3 \%$ for every increase of one thousand Euros in the GDP per capita and increased by $5 \%$ for every point increase in income inequality (Gini).

Separate linear and logistic analyses for the 6-10, 1114, and 15-17 years child age groups revealed slightly larger effects of parental occupational status for the older groups. The effect of national wealth was strongest for ages 11-14. In female reporting parents, low occupational status was associated with a 1.2 point decrease on the KIDSCREEN-10. For male reporting parents, a decrease of 1.8 points emerged. 
TABLE 2: Child QoL differences between EU 27 countries.

\begin{tabular}{|c|c|c|c|c|}
\hline & & & CREEN-10 & \\
\hline & Mean & $\mathrm{SD}$ & Noticeably low \% & Rasch item fit ${ }^{* *}$ \\
\hline Belgium & 50.20 & 10.60 & 11.96 & $0.92-1.11$ \\
\hline Bulgaria & 52.69 & 8.79 & 5.68 & $0.82-1.12$ \\
\hline Czech Rep. & 48.60 & 10.98 & 16.16 & $0.83-1.18$ \\
\hline Denmark & 48.82 & 9.68 & 13.51 & $0.90-1.15$ \\
\hline Germany & 52.77 & 8.96 & 5.27 & $0.79-1.08$ \\
\hline Estonia & 43.06 & 9.23 & 29.50 & $0.87-1.17$ \\
\hline Greece & 49.42 & 8.48 & 8.15 & $0.85-1.11$ \\
\hline Spain & 51.26 & 9.16 & 7.60 & $0.92-1.12$ \\
\hline France & 45.10 & 10.11 & 23.43 & $0.92-1.13$ \\
\hline Ireland & 52.68 & 8.59 & 5.35 & $0.82-1.13$ \\
\hline Italy & 49.73 & 8.79 & 9.38 & $0.95-1.20$ \\
\hline Cyprus & 49.56 & 9.18 & 12.03 & $0.90-1.15$ \\
\hline Latvia & 44.01 & 9.43 & 24.72 & $0.83-1.15$ \\
\hline Lithuania & 43.25 & 9.72 & 27.39 & $0.70-1.08$ \\
\hline Luxembourg & 53.54 & 8.50 & 4.58 & $0.91-1.02$ \\
\hline Hungary & 51.53 & 9.66 & 9.29 & $0.96-1.17$ \\
\hline Malta & 47.73 & 8.34 & 10.36 & $0.79-1.21$ \\
\hline The Netherlands & 57.92 & 9.07 & 1.92 & $1.03-1.31$ \\
\hline Austria & 56.04 & 8.64 & 3.24 & $0.91-1.17$ \\
\hline Poland & 47.55 & 8.89 & 15.11 & $0.85-1.15$ \\
\hline Portugal & 52.23 & 9.97 & 8.94 & $0.84-1.24$ \\
\hline Romania & 48.05 & 9.79 & 14.95 & $0.80-1.12$ \\
\hline Slovenia & 50.71 & 10.05 & 10.13 & $0.88-1.21$ \\
\hline Slovakia & 47.62 & 11.37 & 18.90 & $0.90-1.14$ \\
\hline Finland & 51.67 & 7.26 & 2.90 & $0.89-1.07$ \\
\hline Sweden & 51.80 & 8.88 & 6.18 & $0.89-1.13$ \\
\hline UK & 51.29 & 9.07 & 8.14 & $0.78-1.11$ \\
\hline All & 50.00 & 10.00 & 11.63 & $0.87-1.13$ \\
\hline
\end{tabular}

** INFIT MSQ: infit mean squares residual, and values between 0.7 and 1.3 denote a good itemfit (Bond and Fox, 2001) [27].

Low educational status of a female reporting parent was associated with a decrease of 1.1 points on the KIDSCREEN-10. In male reporting parents this association was not statistically significant.

\section{Discussion}

Our results confirmed previous findings [5, 18, 21, 32]. Taking into account the content of the applied KIDSCREEN-10 measure, children and adolescents from countries with lower GDP and larger income inequalities, whose parents had a lower occupational status and lower educational status, are at higher risk for the following: to feel sad or lonely; to feel less fit and well or full of energy; to be less likely to get on well at school or to be able to concentrate; to be less likely to have enough time for themselves or to do the things that they want to do; to have more often felt treated unfair by their parents; and to less often have fun with friends. Studies using HBSC data also showed higher levels of health complaints with increasing income inequality in a country [33]. While at macro level, an association between national income and life satisfaction could be found, at individual level the effect of GDP and Gini depended on the individual family affluence status [34]. Ottova et al. [35] found a lower risk for health complaints in countries with higher HDI.

4.1. Macroeconomic Factors. We found large cross-national variation in children's QoL and wellbeing. A sizeable portion of this variability was attributable to aspects of national wealth. National wealth may reflect the following factors: economic resources available to individual households; public sector spending or investments in medical technology and health care; improved access to nutrition, goods, and transportation; and investments in education and social protection [36]. Future research is warranted to unravel the particular importance of these factors in explaining the differences found in the present study.

4.2. Microeconomic Factors. Low occupational and low educational status of the responding parent was associated with lower QoL and wellbeing ratings within all countries under study. Interestingly, we found the effect of parental SES to be 
TABLE 3: Regression of KIDSCREEN-10 Index scores on sociodemographic and socioeconomic factors: a multilevel model analysis across countries using random intercepts and random slopes.

\begin{tabular}{|c|c|c|c|c|c|c|}
\hline & \multicolumn{6}{|c|}{ KIDSCREEN-10 score } \\
\hline & Point estimate $\beta$ & $95 \% \mathrm{CI}$ & VPC & $P(\mathrm{VPC})$ & ICC & $95 \%$ population variation \\
\hline Intercept & 52.26 & [51.06-53.45] & 0.13 & $<0.001$ & 0.74 & {$[46.65-57.87]$} \\
\hline Intercept on GDP ${ }^{\#}$ & 0.09 & [0.03-0.15] & - & - & - & \\
\hline 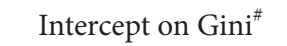 & -0.22 & {$[-0.42--0.01]$} & - & - & - & \\
\hline Boy $6-10^{\# \#}$ & & - & & & & \\
\hline Boy 11-14 & -1.65 & {$[-2.09--1.22]$} & $<0.01$ & $>0.500$ & 0.14 & {$[-2.08--1.22]$} \\
\hline Boy $15-17$ & -2.80 & {$[-3.30--2.30]$} & $<0.01$ & $>0.500$ & 0.13 & {$[-3.23--2.36]$} \\
\hline Girl 6-10 & 0.66 & [0.19-1.14] & 0.01 & $>0.500$ & 0.17 & [0.13-1.20] \\
\hline Girl 11-14 & -1.56 & {$[-2.09--1.03]$} & $<0.01$ & $>0.500$ & 0.14 & {$[-2.00--1.14]$} \\
\hline Girl 15-17 & -2.47 & {$[-3.22--1.73]$} & 0.02 & 0.020 & 0.43 & {$[-4.28--0.66]$} \\
\hline \multicolumn{7}{|l|}{ Occupation high $\#$ a } \\
\hline Occupation medium ${ }^{\mathrm{b}}$ & -0.45 & {$[-0.84--0.06]$} & $<0.01$ & $>0.500$ & 0.13 & {$[-0.83--0.05]$} \\
\hline Occupation low ${ }^{c}$ & -1.44 & {$[-2.08--0.80]$} & 0.01 & $>0.500$ & 0.14 & {$[-2.13--0.81]$} \\
\hline \multicolumn{7}{|l|}{ Education normal $^{\# \mathrm{~d}}$} \\
\hline Education low ${ }^{e}$ & -0.81 & {$[-1.29--0.32]$} & 0.01 & 0.238 & 0.27 & {$[-1.58-0.02]$} \\
\hline \multicolumn{7}{|l|}{ Male report. parent ${ }^{\# \#}$} \\
\hline Female report. parent & -0.60 & {$[-1.06--0.14]$} & 0.01 & 0.036 & 0.40 & {$[-1.67-0.44]$} \\
\hline
\end{tabular}

${ }^{\#}$ Regression of random intercept/slope on second level aggregate socioeconomic characteristics; ${ }^{\# \#}$ reference category.

${ }^{a}$ Professional lawyer, medical practitioner, accountant, architect, general and top management, or manager of company.

${ }^{b}$ Owner of a shop, craftsman, or other.

${ }^{c}$ Farmer, forester, fisherman, manual worker, or unskilled manual worker.

${ }^{\mathrm{d}}$ Finished full-time education at age 17 years and older.

${ }^{\mathrm{e}}$ Finished full-time education at age below 17 years or never been in full-time education.

$\beta$ : raw regression coefficient; VPC: variance partitioning coefficient.

TABLE 4: Regression of noticeably low KIDSCREEN-10 scores on sociodemographic and socioeconomic factors: a multilevel model analysis across countries using random intercepts and random slopes.

\begin{tabular}{|c|c|c|c|}
\hline & \multicolumn{3}{|c|}{ Noticeably low KIDSCREEN-10 results } \\
\hline & Point estimate OR & $95 \% \mathrm{CI}$ & $95 \%$ population variation \\
\hline Intercept & 0.05 & {$[0.03-0.08]$} & {$[0.02-0.16]$} \\
\hline Intercept on GDP $\mathrm{GP}^{\#}$ & 0.97 & {$[0.96-0.99]^{\mathrm{e}}$} & - \\
\hline Intercept on $\mathrm{Gini}^{\#}$ & 1.05 & {$[1.01-1.10]$} & - \\
\hline \multicolumn{4}{|l|}{ Boy $6-10^{\# \#}$} \\
\hline Boy 11-14 & 1.79 & {$[1.48-2.18]$} & {$[1.54-2.08]$} \\
\hline Boy $15-17$ & 2.23 & {$[1.84-2.70]$} & {$[1.99-2.50]$} \\
\hline Girl 6-10 & 0.93 & {$[0.77-1.12]$} & {$[0.81-1.06]$} \\
\hline Girl 11-14 & 1.53 & {$[1.25-1.87]$} & [1.24-1.89] \\
\hline Girl 15-17 & 2.00 & {$[1.51-2.64]$} & {$[1.12-3.57]$} \\
\hline \multicolumn{4}{|l|}{ Occupation high ${ }^{\# \# a}$} \\
\hline Occupation medium $^{\mathrm{b}}$ & 1.23 & {$[1.02-1.48]$} & {$[1.02-1.47]$} \\
\hline Occupation low ${ }^{c}$ & 1.43 & {$[1.16-1.77]$} & [1.21-1.69] \\
\hline \multicolumn{4}{|l|}{ Education normal ${ }^{\# d}$} \\
\hline Education low ${ }^{\mathrm{e}}$ & 1.28 & [1.09-1.49] & [1.11-1.47] \\
\hline \multicolumn{4}{|l|}{ Male report. parent } \\
\hline Female report. parent & 1.14 & {$[0.99-1.32]$} & {$[0.96-1.36]$} \\
\hline
\end{tabular}

\#Regression of random intercept/slope on second level aggregate socioeconomic characteristics; ${ }^{\# \#}$ reference category.

${ }^{a}$ Professional lawyer, medical practitioner, accountant, architect, general and top management, or manager of company.

${ }^{\mathrm{b}}$ Owner of a shop, craftsman, or other.

${ }^{\mathrm{c}}$ Farmer, forester, fisherman, manual worker, or unskilled manual worker

${ }^{\mathrm{d}}$ Finished full-time education at age 17 years and older.

${ }^{\mathrm{e}}$ Finished full-time education at age below 17 years or never been in full-time education.

OR: odds ratio. 
stable across countries, whereas in the HBSC study a variation in the strength of this association appeared [23]. Families with high incomes may be able to provide their children with more goods, services, and resources that can benefit their children and prevent them from experiencing adverse QoL [37]. von Rueden et al. [5] showed that children and adolescents with better access to the following factors report a higher QoL: places for social, cultural, educational, or other purposes; the availability of transportation like a car; an unshared bedroom (i.e., privacy); family holidays (i.e., experiencing different cultures); and media.

Different studies found that some determinants-such as parent mental health, having many children in the household, and the availability of external support systems-appear to have a mediating effect between low SES and poor child health $[38,39]$. Lack of stable income, poor parental selfesteem related to unemployment, and lack of social and community supports for parents may modify parents' abilities to provide optimal and consistent parenting [40]. This mechanism may contribute to higher rates of emotional and behavioural problems in children [38].

The separate analyses across age groups do not support other studies showing that, with growing independence, the influence of parental socioeconomic status is reduced and peers become a more important reference group [8]. However, it is possible that a parental QoL and wellbeing rating for social equalisation affects this more than adolescents' selfreported QoL and wellbeing.

The SES effects that we found in our study are rather small. Though their children experience more psychosocial and emotional problems, parents in economically poorer families where lack of material resources is the norm may accept problems of family members as the rule rather than the exception, and thus they might not consider that as having an adverse impact on social functioning or daily activities [41].

Parental proxy reports of their children's QoL and wellbeing should be considered carefully as a potential substitute for self-reports [42]. However, it is widely recognised that both, self-reports and proxy reports, should constitute important complementary information $[43,44]$, as proxy reports provide at least a partial view of a child's QoL and wellbeing [45], complemented by important additional information from parents.

Finally, it should also be mentioned that the main outcome in our study, QoL and wellbeing, is generally considered to be a multidimensional construct [46]. According to some authors, however, it can also be measured using summary measures yielding in a single, overall score of QoL [47-49], as was done in our study. In fact, the type of assessment we employed in this study is quite common in current measurement practices of QoL and wellbeing.

4.3. Strengths of the Study. The strengths of this study are both the use of the KIDSCREEN-10 measure that was developed and confirmed for cross-cultural psychometric functioning and the fact that it is composed of indicators which are accepted, relevant, and understandable in a comparable manner across different countries while also providing interval-scaled information [24]. Thus, in contrast to other studies, it is likely that any differences found between countries are attributable to "true" cross-national differences and not a differential functioning of the measure across countries.

4.4. Limitations of the Study. The primary limitation is the fact that we assessed occupational status of the responding parent (mainly mothers), which is likely to be lower than that of the second adult in the household. Interestingly, male parental occupational status was found to be more important for children's QoL and wellbeing than female parental occupational status. Conversely, only female parental educational status was of importance for children's wellbeing.

We did not study additional SES aspects, such as familial material welfare, income, housing conditions, and socioeconomic status of the neighbourhood. Restricting SES to parental occupational and educational status might explain why only small individual level effect sizes have been observed in this study.

The actual analysis of cross-sectional data precludes causal interpretation, for example, regarding the extent that economic growth could lead to improvements in population QoL and wellbeing [50-52]. Also, the possibility of reverse causation (i.e., having a sick child affecting employment and income) cannot be dismissed in this type of study.

\section{Outlook}

Our study hinted at the potential benefits that increasing national wealth, while simultaneously decreasing income inequality, might have for health of individuals in a society (or in societies in general). However, as this is generally difficult to achieve, further research is needed to identify those mechanisms linking low SES with low QoL and poorer wellbeing. Preventive public health actions could then focus on these mechanisms. For the promotion of health, it might be important to consider not only how much money there is available (in a society) but-maybe even more importantlyto what socially productive ends it is devoted. To reduce avoidable health differences, it is important that different social groups (e.g., migrants, single-parent families, or unemployed households) are able to access and benefit from such investments.

What Is Already Known on This Topic

(i) Large European cross-national differences in children and adolescents' self-reported wellbeing and QoL have been observed.

(ii) A substantial proportion of these differences are associated with differences in national wealth and income inequality.

(iii) Social inequalities in adolescents' QoL within countries have been observed for some European countries only. 
What This Study Adds

(i) The study shows large cross-national differences in children and adolescents' QoL and wellbeing and their association with national wealth and income inequality.

(ii) The applied interval-scaled and cross-national comparable QoL measure enabled a precise and valid estimation of the magnitude of within and between country differences in children and adolescents' QoL and wellbeing that are attributable to wealth and SES.

(iii) The analyses showed that children's QoL reported by parents covaried with national wealth and socioeconomic status at individual level.

(iv) Using an interval-scaled and cross-culturally comparable QoL measure revealed a stable pattern of association between parental SES and their children's QoL and wellbeing for each EU 27 countries.

\section{Acknowledgments}

The Flash Eurobarometer Survey no. 246 was conducted by the Gallup Organization. The authors thank all coworkers of this scientific unit for their contribution and also all families who participated in the survey. The authors would also like to acknowledge Jürgen Scheftlein and thank him for his support of the survey. The Flash Eurobarometer Survey no. 246 was carried out at the request of the European Commission, Health and Consumer DG, and C4-Health Determinants. The writing and submission of the paper was not contingent on the approval or censorship of the European Commission. There was no direct financial support for the preparation of the paper. Basic descriptive results (not reported in this paper) were reported by the European Commission elsewhere. The authors declare no financial disclosure and no conflicting interests associated with the paper.

\section{References}

[1] J. W. Lynch and G. Kaplan, "Socioeconomic position," in Social Epidemiology, L. F. Berkman and I. Kawachi, Eds., pp. 13-25, Oxford University Press, New York, NY, USA, 2000.

[2] M. Richter, M. Erhart, C. A. Vereecken, A. Zambon, W. Boyce, and S. N. Gabhainn, "The role of behavioural factors in explaining socio-economic differences in adolescent health: a multilevel study in 33 countries," Social Science and Medicine, vol. 69, no. 3, pp. 396-403, 2009.

[3] E. Chen, K. A. Matthews, and W. T. Boyce, "Socioeconomic differences in children's health: how and why do these relationships change with age?" Psychological Bulletin, vol. 128, no. 2, pp. 295329, 2002.

[4] N. J. Spencer, "Social equalization in youth: evidence from a cross-sectional British survey," European Journal of Public Health, vol. 16, no. 4, pp. 368-375, 2006.

[5] U. von Rueden, A. Gosch, L. Rajmil, C. Bisegger, and U. RavensSieberer, "Socioeconomic determinants of health related quality of life in childhood and adolescence: results from a European study," Journal of Epidemiology and Community Health, vol. 60, no. 2, pp. 130-135, 2006.

[6] E. Goodman, "The role of socioeconomic status gradients in explaining differences in US adolescents' health," American Journal of Public Health, vol. 89, no. 10, pp. 1522-1528, 1999.

[7] M. Halldorsson, A. E. Kunst, L. Kohler, and J. P. Mackenbach, "Socioeconomic inequalities in the health of children and adolescents: a comparative study of the five Nordic countries," European Journal of Public Health, vol. 10, no. 4, pp. 281-288, 2000.

[8] P. West, "Health inequalities in the early years: is there equalisation in youth?" Social Science and Medicine, vol. 44, no. 6, pp. 833-858, 1997.

[9] B. Starfield, A. W. Riley, W. P. Witt, and J. Robertson, "Social class gradients in health during adolescence," Journal of Epidemiology and Community Health, vol. 56, no. 5, pp. 354-361, 2002.

[10] R. H. Bradley and R. F. Corwyn, "Socioeconomic status and child development," Annual Review of Psychology, vol. 53, pp. 371-399, 2002.

[11] A. Leplège and S. Hunt, "The problem of quality of life in medicine," The Journal of the American Medical Association, vol. 278, pp. 47-50, 1997.

[12] M. Seid, J. W. Varni, D. Segall, and P. S. Kurtin, "Health-related quality of life as a predictor of pediatric healthcare costs: a twoyear prospective cohort analysis," Health and Quality of Life Outcomes, vol. 2, article 48, 2004.

[13] J. W. Varni, T. M. Burwinkle, and M. M. Lane, "Health-related quality of life measurement in pediatric clinical practice: an appraisal and precept for future research and application," Health and Quality of Life Outcomes, vol. 3, article 34, 2005.

[14] Committee on Evaluation of Children's Health NRC, "Children's Health, the Nation's Wealth: Assessing and Improving Child Health," National Academies Press, Washington, DC, USA ,2004.

[15] W. Kuyken, "The World Health Organization Quality of Life Assessment (WHOQOL): position paper from the World Health Organization," Social Science and Medicine, vol. 41, no. 10, pp. 1403-1409, 1995.

[16] E. Diener, B. Diener, and R. Will, "Money increase subjective well-being? a literature review and guide to needed research," in the Science of Well-Being: The Collected Work of Ed Diener, E. Diener, Ed., pp. 119-154, Springer, Dordrecht, The Netherlands, 2009.

[17] K. E. Pickett and M. Pearl, "Multilevel analyses of neighbourhood socioeconomic context and health outcomes: a critical review," Journal of Epidemiology and Community Health, vol. 55, no. 2, pp. 111-122, 2001.

[18] T. Torsheim, C. Currie, W. Boyce, I. Kalnins, M. Overpeck, and S. Haugland, "Material deprivation and self-rated health: a multilevel study of adolescents from 22 European and North American countries," Social Science and Medicine, vol. 59, no. 1, pp. 1-12, 2004.

[19] R. G. Wilkinson, "Socioeconomic determinants of health: health inequalities: relative or absolute material standards?" British Medical Journal, vol. 314, no. 7080, pp. 591-595, 1997.

[20] J. Lynch, G. D. Smith, M. Hillemeier, M. Shaw, T. Raghunathan, and G. Kaplan, "Income inequality, the psychosocial environment, and health: comparisons of wealthy nations," The Lancet, vol. 358 , no. 9277, pp. 194-200, 2001. 
[21] T. Torsheim, C. Currie, W. Boyce et al., "Stronger reference here somewhere to absolute and relative health inequalities!," Journal of Epidemiology \& Community Health, vol. 60, pp. 156-161, 2006.

[22] T. A. B. Snijders, Multilevel Analysis: An Introduction to Basic and advAnced Multilevel Modeling, Sage, London, UK, 1999.

[23] A. Zambon, W. Boyce, E. Cois et al., "Do welfare regimes mediate the effect of socioeconomic position on health in adolescence? A Cross-national comparison in Europe, North America, and Israel," International Journal of Health Services, vol. 36, no. 2, pp. 309-329, 2006.

[24] U. Ravens-Sieberer, “The KIDSCREEN questionnaires. Quality of life questionnaires for children and adolescents-handbook," Papst Science Publisher, Lengerich, Germany, 2006.

[25] U. Ravens-Sieberer, M. Erhart, L. Rajmil et al., "Reliability, construct and criterion validity of the KIDSCREEN-10 score: a short measure for children and adolescents' well-being and health-related quality of life," Quality of Life Research, vol. 19, no. 10, pp. 1487-1500, 2010.

[26] Gallup Organization, "Parent's views on the mental health of their child. Analytical report," Flash Eurobarometer Report 246, European Commision, DG Communication, Public Opinion, Brussels, Belgium, 2009.

[27] T. G. Bond and C. M. Fox, Applying the Rasch Model: Fundamental Measurement in the Human Sciences, Taylor \& Francis, Mahwah, NJ, USA, 2001.

[28] Directorate of Intelligence, The World Factbook, Central Intelligence Agency, Washington, DC, USA, 2009.

[29] World Bank, World Development Indicators 2007, World Bank, Washington, DC, USA, 2007.

[30] S. W. Raudenbush, A. S. Bryk, and R. Congdon, HLM For Windows 5.05, Scientific Software International, Lincolnwood, Ill, USA, 2001.

[31] J. Cohen, Statistical Power Analysis for the Behavioural Sciences, Erlbaum, New York, NY, USA, 1988.

[32] J. P. Mackenbach, Health Inequalities: Europe in Profile. An Independent Expert Report Commissioned By the UK Presidency of the EU, Department of Health, London, UK, 2006.

[33] B. E. Holstein, C. Currie, W. Boyce et al., "Socio-economic inequality in multiple health complaints among adolescents. International comparative study in 37 countries," International Journal of Public Health, vol. 54, no. 2, supplement, pp. S260S270, 2009.

[34] K. A. Levin, T. Torsheim, W. Vollebergh et al., "National income and income inequality, family affluence and life satisfaction among 13 year old boys and girls: a multilevel study in 35 countries," Social Indicators Research, vol. 104, no. 2, pp. 179194, 2011.

[35] V. Ottova, M. Erhart, W. Vollebergh et al., "The role of individual and macro-level social determinants on young adolescents' psychosomatic complaints," Journal of Early Adolescence, vol. 32, no. 1, pp. 126-158, 2012.

[36] S. Taylor, "Wealth, health and equity: convergence to divergence in late 20th century globalization," British Medical Bulletin, vol. 91, no. 1, pp. 29-48, 2009.

[37] J. Brooks-Gunn and G. J. Duncan, "The effects of poverty on children," Future of Children, vol. 7, no. 2, pp. 55-71, 1997.

[38] C. C. Victorino and A. H. Gauthier, "The social determinants of child health: variations across health outcomes-a populationbased cross-sectional analysis," BMC Pediatrics, vol. 9, article 53, 2009.
[39] N. Garmezy, "Children in poverty: resilience despite risk," Psychiatry, vol. 56, no. 1, pp. 127-136, 1993.

[40] E. L. Schor and E. G. Menaghan, "Family pathways to child health," in Society and Health, B. C. Amick, S. Levine, A. R. Tarlov, and D. Walsh, Eds., pp. 18-45, Oxford University Press, 1995.

[41] N. J. Spurrier, M. G. Sawyer, J. J. Clark, and P. Baghurst, "Socioeconomic differentials in the health-related quality of life of Australian children: results of a national study," Australian and New Zealand Journal of Public Health, vol. 27, no. 1, pp. 27-33, 2003.

[42] A. Jokovic, D. Locker, and G. Guyatt, "How well do parents know their children? Implications for proxy reporting of child health-related quality of life," Quality of Life Research, vol. 13, no. 7, pp. 1297-1307, 2004.

[43] C. Eiser and R. Morse, "Quality-of-life measures in chronic diseases of childhood," Health Technology Assessment, vol. 5, no. 4, pp. 1-147, 2001.

[44] M. De Civita, D. Regier, A. H. Alamgir, A. H. Anis, M. J. FitzGerald, and C. A. Marra, "Evaluating health-related qualityof-life studies in paediatric populations: some conceptual, methodological and developmental considerations and recent applications," PharmacoEconomics, vol. 23, no. 7, pp. 659-685, 2005.

[45] M. White-Koning, C. Arnaud, S. Bourdet-Loubère et al., "Subjective quality of life in children with intellectual impairmenthow can it be assessed?" Developmental Medicine and Child Neurology, vol. 47, no. 4, pp. 281-285, 2005.

[46] U. Ravens-Sieberer, M. Erhart, N. Wille, R. Wetzel, J. Nickel, and M. Bullinger, "Generic health-related quality-of-life assessment in children and adolescents: methodological considerations," PharmacoEconomics, vol. 24, no. 12, pp. 1199-1220, 2006.

[47] M. Bullinger, "Forschungsinstrumente zur Erfassung der Lebensqualität bei Krebs-ein Überblick," in Psychosoziale Aspekte der Krebsforschung (Psychosoziale Onkologie) Jahrbuch der Medizinischen Psychologie, B. Verres and M. Hasenbring, Eds., pp. 45-57, Springer, Berlin, Germany, 3rd edition, 1989.

[48] R. J. Rogerson, "Environmental and health-related quality of life: conceptual and methodological similarities," Social Science and Medicine, vol. 41, no. 10, pp. 1373-1382, 1995.

[49] M. Herdman, L. Rajmil, U. Ravens-Sieberer et al., "Expert consensus in the development of a European health-related quality of life measure for children and adolescents: a Delphi study," Acta Paediatrica, vol. 91, no. 12, pp. 1385-1390, 2002.

[50] R. A. Easterlin, "Explaining happiness," Proceedings of the National Academy of Sciences of the United States of America, vol. 100, no. 19, pp. 11176-11183, 2003.

[51] J. Lynch, G. D. Smith, S. Harper et al., "Is income inequality a determinant of population health? Part 1. A systematic review," Milbank Quarterly, vol. 82, no. 1, pp. 5-99, 2004.

[52] M. R. Hagerty and R. Veenhoven, "Wealth and happiness revisited-growing national income does go with greater happiness," Social Indicators Research, vol. 64, no. 1, pp. 1-27, 2003. 


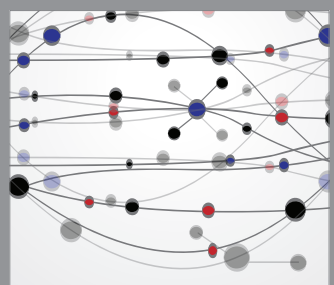

The Scientific World Journal
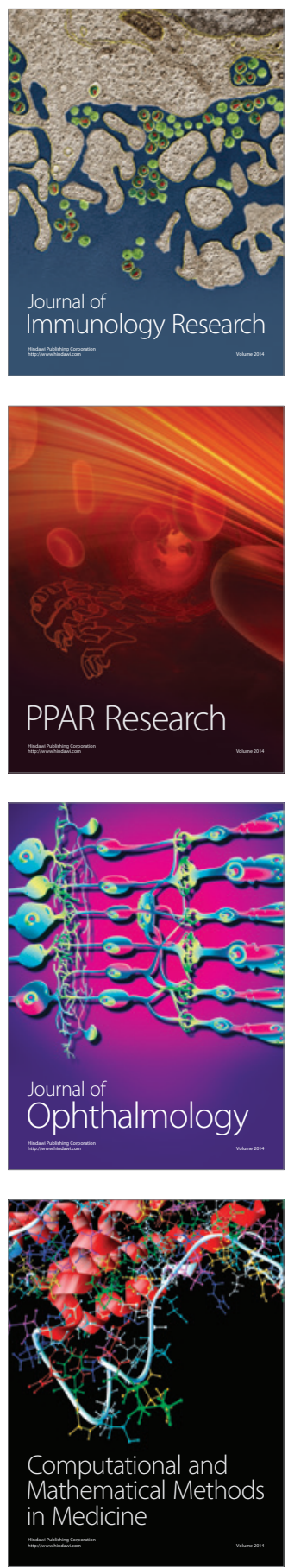

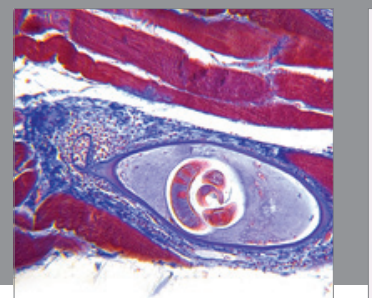

Gastroenterology

Research and Practice
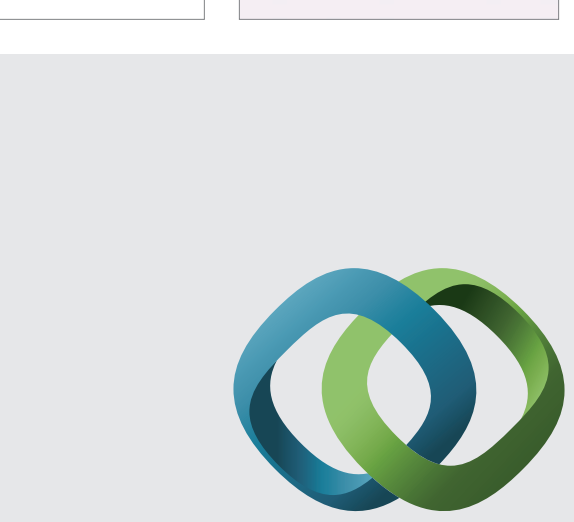

\section{Hindawi}

Submit your manuscripts at

http://www.hindawi.com
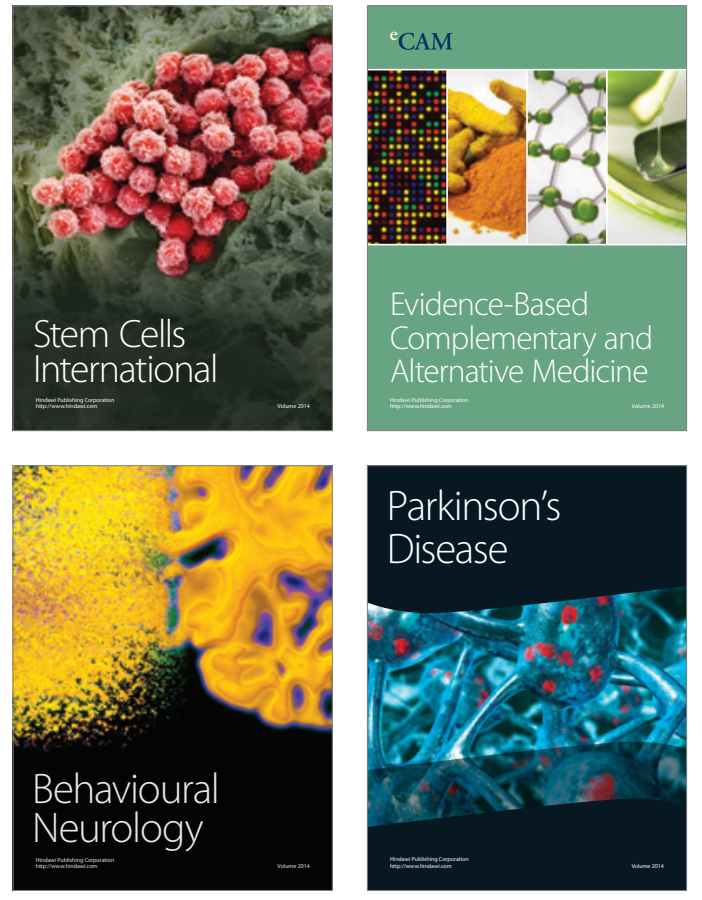
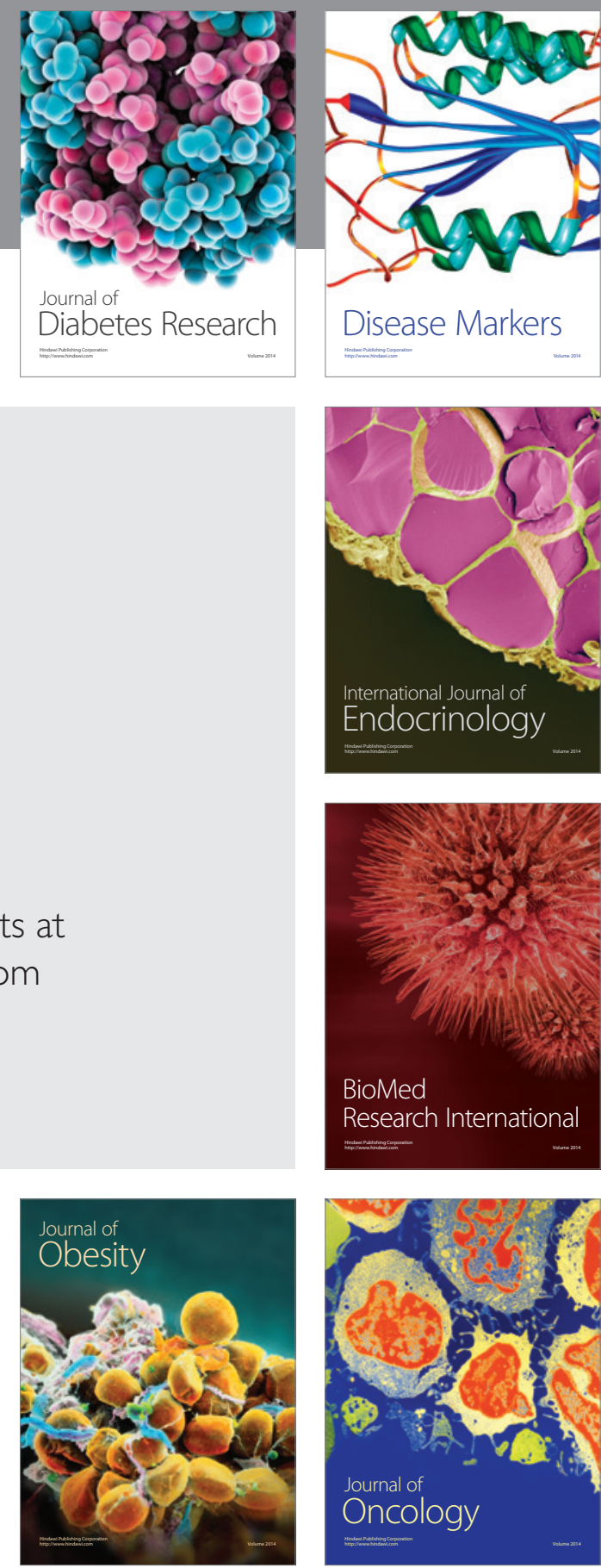

Disease Markers
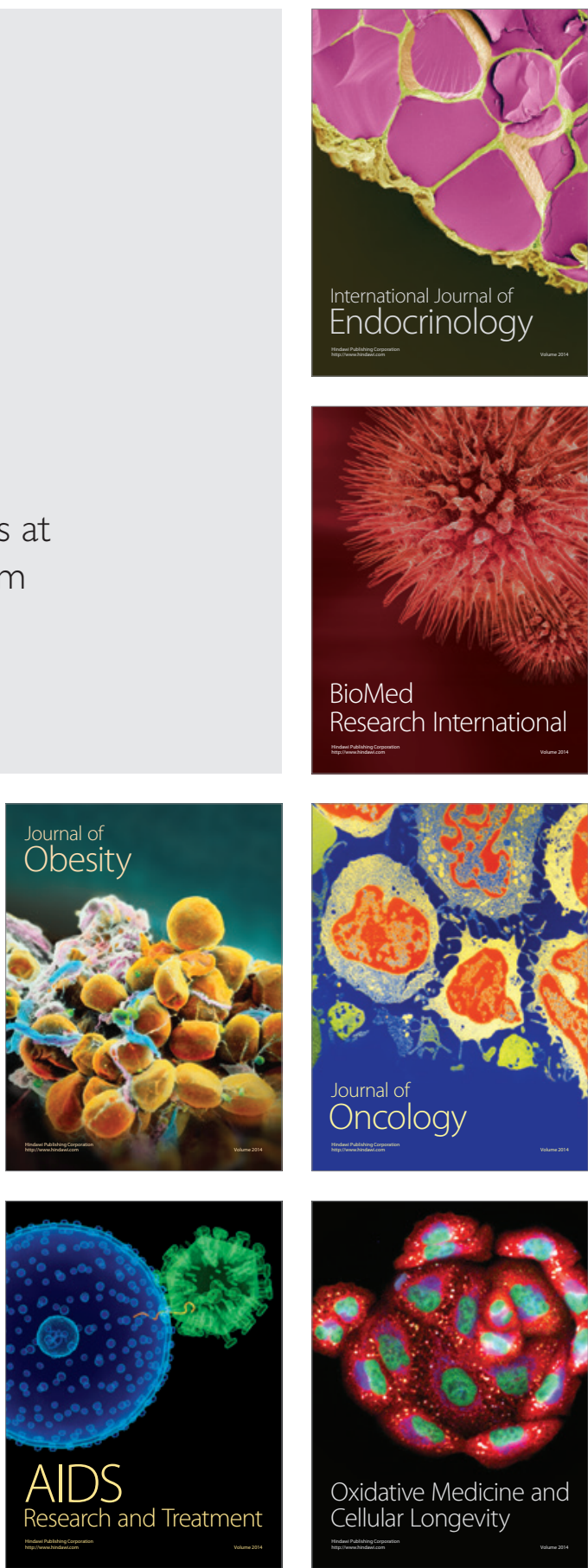